\title{
Bicuspid aortic stenosis in transcatheter aortic valve replacement era: Emerging confusions hindering the standardization of the procedure
}

\author{
Tian-Yuan Xiong, Zhen-Gang Zhao, Mao Chen \\ Department of Cardiology, West China Hospital, Sichuan University, Chengdu, China
}

Bicuspid aortic valve (BAV) has long been regarded as a relative contraindication for transcatheter aortic valve replacement (TAVR). The incidence of aortic stenosis in patients with BAV is high, but the onset of symptoms is usually at a relatively young age. As TAVR is moving to younger and lower-risk patients, the proportion of patients with BAV will increase. Some initial off-label experience and small registries have demonstrated the safety and efficacy of treating bicuspid aortic stenosis with TAVR. Novel strategies dealing with $\mathrm{BAV}$ and devices are also emerging along the way aiming for better outcomes. Nevertheless, multicenter collaborative randomized controlled trials are required before official approval in this field. For this purpose, it is time to clarify several confusions that hinders the standardization of the procedure in BAV (Table 1).

Not all BAV subtypes behave the same or are suitable for TAVR. However, there is no consensus regarding which anatomical presentation should be selected for the procedure. The current classification system of BAV does not satisfy the need for TAVR and only provides limited information for patient selection and prediction of outcomes. In a recent attempt to simplify BAV classification relevant to TAVR, a previously neglected morphology of tricommissural (functional/acquired) BAV was delineated [1]. However, the proposed system failed to predict the rates of moderate or severe aortic regurgitation at discharge or the new permanent pacemakers at 30 days.

Classification for BAV in the TAVR era is indeed difficult due to the involvement of many potential factors that could influence outcomes.
A better understanding of the calcification mode and its behavior is required for an evaluation of procedural feasibility and risk in BAV. Leaflet length and bulkiness relative to sinus size, in addition to coronary height should be assessed, to better predict coronary risk. Thus, apart from the number of cusps and commissures and modes of fusions, raphe length, calcification modes and its distribution, leaflet length, size and asymmetry of sinuses may also need to be incorporated into a future system.

It has been shown that new-generation valves reduced the rates of paravalvular leak (PVL), annular rupture and second valve implantation compared with the early-generation ones [2]. Despite having the PVL issue being tackled with an additional seal on the prosthesis, the common noncircular shape of prosthesis frame after deployment observed in BAV still raises concerns for durability/bioprosthesis degeneration when considering the younger ages of BAV patients being referred, especially with the intra-annular valve design.

A BAV-friendly prosthesis design, takes advantage of both from the current balloon-expandable and self-expanding devices, which could be a potential solution. The proposed design should ideally a) have a strong radial force to circularize the native anatomy and avoid severe stent deformation; b) be supra-annular, so as to utilize the stent frame below the nadir of bioprosthesis as part of left ventricular outflow tract to achieve better geometry at the level of the functioning leaflets and larger effective orifice area; c) be relatively short to prevent radial force being exposed to possible untreated aortic aneurysm.

Address for correspondence: Mao Chen, MD, PhD, Department of Cardiology, West China Hospital, Sichuan University, \#37 Guo Xue Alley, Chengdu, 610041, PR China, tel: 86-28-85423362, fax: 86-28-85423170, e-mail: hmaochen@vip.sina.com 
Table 1. Summary of confusions that hinders the standardization of transcatheter aortic valve replacement (TAVR) in bicuspid aortic valve (BAV).

\begin{tabular}{lll}
\hline Confusions & Importance of clarifying the problem & Possible solutions \\
\hline Anatomical classification & $\begin{array}{l}\text { Current classification system of BAV } \\
\text { could not satisfy TAVR need and only } \\
\text { provides limited information for patient } \\
\text { selection and the prediction of outcomes }\end{array}$ & $\begin{array}{l}\text { Apart from the number of cusps and } \\
\text { commissures and modes of fusions, } \\
\text { raphe length, calcification modes and } \\
\text { its distribution, leaflet length, size and } \\
\text { asymmetry of sinuses may also need } \\
\text { to be incorporated into a future system }\end{array}$ \\
$\begin{array}{lll}\text { BAV-friendly } \\
\text { prosthesis design }\end{array}$ & $\begin{array}{l}\text { The common non-circular shape of } \\
\text { implanted stent frame raises the concern } \\
\text { of early bioprosthesis degeneration }\end{array}$ & $\begin{array}{l}\text { Prosthesis design should ideally: a) have } \\
\text { strong radial force; b) be supra-annular; } \\
\text { BAV-specific prosthesis belatively short }\end{array}$ \\
sizing strategy & $\begin{array}{l}\text { Current sizing strategy was developed } \\
\text { from tricuspid aortic valve, which does } \\
\text { not fully appreciate the 'fish-mouth' } \\
\text { valvular opening often seen in BAV } \\
\text { and may result in excessive oversizing }\end{array}$ & $\begin{array}{l}\text { To predict the anchoring zone accurately, } \\
\text { which relies on investigations on the } \\
\text { behavior of BAV leaflets and transcatheter } \\
\text { heart valve stent based on post-implant } \\
\text { computed tomography, mechanics and } \\
\text { flow field, and the development of } \\
\text { dedicated software }\end{array}$ \\
& & $\begin{array}{l}\text { One-stage transcatheter treatment } \\
\text { of bicuspid aortic stenosis with } \\
\text { concomitant aortopathy }\end{array}$ \\
\hline
\end{tabular}

Selection of the right prosthesis size is of great importance to the success of TAVR. The fact that perimeter- or area-derived diameters at the annulus are usually much larger than that of the exact valvar opening (typical 'fish-mouth' appearance) in BAV [3] makes the conventional sizing strategy based on the annulus less appealing. Thus, some novel strategies appear. As a preparatory step, balloon aortic valvuloplasty enables a direct view of the specific anatomical characteristics and post-implant prediction [4]. However, due to the different shape and length of a balloon compared to a certain device, the prediction from balloon-sizing will be imperfect. Another theory is supra-annular sizing. The spline was determined between 4 and $8 \mathrm{~mm}$ above the annulus by empirically predicting native cusp capacity for implanted transcatheter heart valve from actual valvar opening, the presence of raphes and calcifications. Similarly, there is the concept of inter-commissural distance, which was measured at $4 \mathrm{~mm}$ above the annulus. The prosthesis inter-commissural distance ratio was found to be smaller than the correspondent prosthesis annulus ratio, suggesting the level $4 \mathrm{~mm}$ above the annulus had more restrictions to the device than the annulus. These latter two concepts are still preliminary, but they define device landing zone besides the annulus by focusing on the region which theoretically restricts the implanted prosthesis most.
The key to a new strategy is an accurate prediction of the anchoring zone. This relies on investigations on the behavior of BAV leaflets and transcatheter heart valve stent based on post-implant computed tomography, mechanics and flow field, and the development of dedicated software to predict the post-implant interactions of stents and leaflets.

The coexistence of an ascending aortic aneurysm is common among patients with bicuspid aortic stenosis, which would render surgery mandatory in order to treat both lesions simultaneously. Isolated TAVR is unable to address aortopathy, which raises the potential risk of rupture or dissection. To date, there have been reports of combined TAVR and thoracic endovascular aneurysm repair.

Although it could be technically challenging, given the trend of developing combined percutaneous solutions to different disease groups, there remains a possibility for a one-stage transcatheter treatment of bicuspid aortic stenosis with concomitant aortopathy by a single device. Benefits of this one-stage procedure would be a reduction in total procedural time, safe valve delivery (within pre-implanted aneurysm stent) and better adaptation to the native anatomy, but issues such as minimizing catheter profile, device length and determining ways of coronary protection etc. need to be resolved first.

Three years ago, there was a review of TAVR utilization in BAV [5]. Back then, the proportion 
of BAV patients was extremely low in fairly large TAVR registries. The myth of denying BAV merely due to this morphology for TAVR has now been partially settled, yet plenty of new uncertainties are revealed. To be an established treatment option for bicuspid aortic stenosis, extensive collaborations between scientists, engineers (valve manufacturer and information technology), imaging specialists, and interventionists are required to develop a new classification system, dedicated software and devices for BAV.

Conflict of interest: None declared

\section{References}

1. Jilaihawi H, Chen M, Webb J, et al. A Bicuspid Aortic Valve Imaging Classification for the TAVR Era. JACC Cardiovasc Im- aging. 2016; 9(10): 1145-1158, doi: 10.1016/j.jcmg.2015.12.022, indexed in Pubmed: 27372022.

2. Yoon SH, Lefèvre T, Ahn JM, et al. Transcatheter aortic valve replacement with early- and new-generation devices in bicuspid aortic valve stenosis. J Am Coll Cardiol. 2016; 68(11): 1195-1205, doi: 10.1016/j.jacc.2016.06.041, indexed in Pubmed: 27609682 .

3. Kochman J, Rymuza B, Huczek Z. Transcatheter aortic valve replacement in bicuspid aortic valve disease. Curr Opin Cardiol. 2015; 30(6): 594-602, doi:10.1097/HCO.0000000000000219, indexed in Pubmed: 26398414.

4. Babaliaros V. To Size or Not to Size - There Is No Question. JACC: Cardiovascular Interventions. 2013; 6(9): 972-973, doi: 10.1016/j.jcin.2013.06.005.

5. Zhao ZG, Jilaihawi H, Feng $\mathrm{Y}$, et al. Transcatheter aortic valve implantation in bicuspid anatomy. Nat Rev Cardiol. 2015; 12(2): 123-128, doi:10.1038/nrcardio.2014.161, indexed in Pubmed: 25311233. 